\section{The Earth's secret companion}

\section{Carl D. Murray}

\section{The Earth has a second companion - an asteroid, about $5 \mathrm{~km}$ across, in a peculiar type of orbital resonance called an overlapping horseshoe. It is probably only a temporary liaison.}

A lthough sharing an orbit with a planet may seem an unlikely way for an asteroid to avoid collisions, being placed at the eye of the dynamical storm does offer some form of protection. The discovery by Wiegert et al. (see page 685 of this issue ${ }^{1}$ ) that the unnamed asteroid 3753 shares its orbit with the Earth is the latest and most unusual example of a co-orbital resonant configuration that has been known for more than two hundred years.

The Italian-French mathematician Joseph-Louis Lagrange (1736-1813) studied the problem of three bodies moving under their mutual newtonian gravitational attraction. In 1772 he showed that a system consisting of the Sun and a planet had two equilibrium points located in the same orbit as the planet, one leading it by $60^{\circ}$ and the other trailing by $60^{\circ}$, each forming an equilateral triangle with the two masses (Fig. 1). Lagrange showed that objects placed at these points were stable to small displacements provided the planet-Sun mass ratio was less than 0.04 , and he predicted that Jupiter might harbour material at its leading and trailing points. It was not until the detection of 588 Achilles in 1906 that Lagrange's prediction was finally verified. There are now known to be several hundred such objects moving around Jupiter's triangular points, referred to as the Trojan asteroids. Not only was the type of orbital behaviour envisaged by Lagrange mathematically possible, but nature had obligingly provided actual physical examples.

Any elliptical orbit is characterized by its eccentricity (the degree of departure from a circle) and its semimajor axis (half the length of the orbit's long axis). Trojan asteroids have semimajor axes that are comparable to Jupiter's, and therefore they are special examples of resonant motion where the orbital periods of both objects are in the approximate ratio of $1: 1$. It is customary to consider the orbital paths of such objects in a frame rotating with the angular velocity of Jupiter. In this frame the Trojan orbits look like tadpoles, with large heads and elongated tails (Fig. 1). At any given time the asteroid is always moving on an ellipse around the Sun, but the orbit slowly evolves as a result of jovian perturbations.

The fact that the trailing and leading

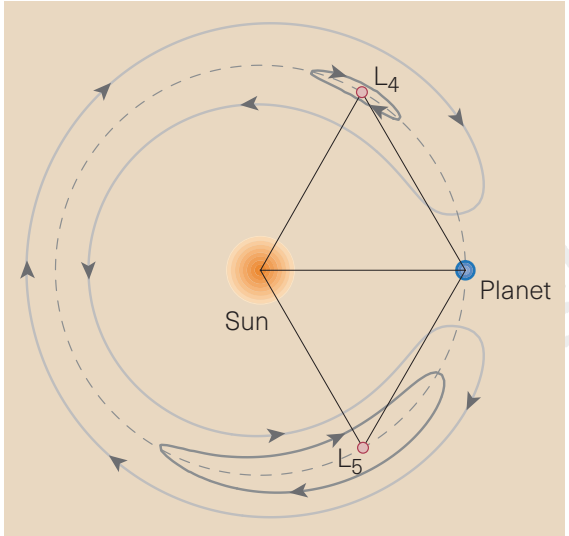

Figure 1 Three resonant orbits in a Sun-planet system, shown in a frame rotating with the planet's orbit. The stable equilibrium point $\mathrm{L}_{4}$ lies ahead of the planet by $60^{\circ}$; the $L_{5}$ point trails. By Kepler's third law, those objects exterior to the planet move more slowly than it, and those interior move faster; that determines the direction of the path in the rotating frame. Here, two tadpole orbits are shown, as is a horseshoe orbit around both stable points. These are the actual paths traced out over many orbital periods in the case where the keplerian orbits are nearly circular. For eccentric orbits each path develops loops, as the object moves around its ellipse over one orbital period. Relative to the Earth, asteroid 3753 moves in an 'overlapping horseshoe' path (see Fig. 1 on page 685). Its orbit can overlap Earth's with no danger of collision because it is in a highly inclined plane.

points are stable for every planet orbiting the Sun and every satellite orbiting a planet (with the exception of the Pluto-Charon system) means that tadpole orbits could exist in other systems. This has proved to be so. In 1990 the asteroid (5261) Eureka was discovered orbiting in a 1:1 resonance near the trailing point of the Sun-Mars system ${ }^{2}$. In Saturn's system, it is known that the moon Tethys has smaller moons orbiting close to each of its triangular points, and Dione shares its orbit with a single moon near its leading equilibrium point.

However, another type of orbit is possible in the 1:1 resonance. For a large enough difference between the semimajor axes of the planet and companion, the companion's orbital path can encompass both equilibri- um points. The resulting path is referred to as a 'horseshoe' orbit (Fig. 1). The first example of a horseshoe configuration was announced in 1980 when Janus and Epimetheus, two newly discovered moons of Saturn, were found to be moving in orbits with a radial separation comparable to their smallest dimension $(\sim 50 \mathrm{~km})$. The mass of Epimetheus is 25 per cent that of Janus and so they are large enough to perturb each other's motion. Every four years they encounter without passing, at which time Janus's semimajor axis changes by $10 \mathrm{~km}$ while that of Epimetheus changes in the other direction by $40 \mathrm{~km}$, the situation reversing itself at the next encounter ${ }^{3}$. Once again, nature provided physical confirmation of a theoretical possibility.

The discovery that asteroid 3753 moves in a horseshoe orbit with respect to Earth could only have been made using the sort of numerical integration of the equations of motion carried out by Wiegert and colleagues ${ }^{1}$. The true nature of its orbit was disguised by two things: the orbit is highly inclined to the plane of the Earth's orbit, and its large eccentricity gives rise to the 'loops' characteristic of eccentric paths in rotating frames (these are a simple consequence of viewing the asteroid's motion around the Sun from a frame rotating with the Earth's angular velocity). But the tell-tale switching of 3753's semimajor axis from one side of $1 \mathrm{AU}$ to the other (that is, from inside to outside Earth's orbit) is a property of a horseshoe orbit that is more difficult to hide; $1 \mathrm{AU}$ is the average distance between the Earth and Sun.

There are no simple guarantees of stability for any of the objects known to be in tadpole or horseshoe orbits, and the future for 3753 looks particularly bleak, with a chaotic orbit that can cross that of the Earth and Venus, while also coming close to the orbits of Mercury and Mars. The typical lifetime of such an object ${ }^{4}$ is expected to be about 100 million years.

But where do co-orbital objects come from and why are they not more prevalent, given that the stability condition is so easily satisfied? Whereas it is likely that 3753 is a temporary occupant of Earth's co-orbital niche, the Trojan asteroids in Jupiter's orbit are probably the tadpole survivors of a larger primordial population that once included some horseshoe orbits. Studies of test particles placed near Saturn's equilibrium points ${ }^{5}$ show that instabilities can arise when jovian perturbations are included. In the case of satellites of the outer planets, co-orbital objects could be fragments of the collisional break-up of an earlier, parent satellite. The dynamics of such processes are also applicable to the study of planetary rings, where embedded satellites that maintain particles in horseshoe orbits have been proposed to account for narrow rings ${ }^{6}$. 
Asteroid 3753 won't hit the Earth soon, and we may well avoid physical contact with this particular 5-km companion indefinitely. Many puzzles remain, however, concerning the origin and evolution of all the co-orbital systems. At least now there is a growing realization among planetary scientists that an improved understanding of these intimate 1:1 relationships is essential to studies ranging from planetary accretion to ring dynamics.
Carl D. Murray is in the Astronomy Unit, Queen Mary and Westfield College, London E1 4NS, UK.

1. Wiegert, P. A., Innanen, K. A. \& Mikkola, S. Nature 387, 685-686 (1997).

2. Mikkola, S., Innanen, K. A., Muinonen, K. \& Bowell, E. Celest. Mech. Dyn. Astron. 58, 53-64 (1993).

3. Nicholson, P. D. et al. Icarus 100, 464-484 (1992).

4. Bottke, W. F. et al. in Hazards due to Comets and Asteroids (ed. Gehrels, T.) 337-357 (Univ. Arizona Press, Tucson, 1994).

5. de la Barre, C. M., Kaula, W. M. \& Varadi, F. Icarus 121, 88-113 (1996).

6. Dermott, S. F., Gold, T. \& Sinclair, A. T. Astron. J. 84, 1225-1234 (1979).

\section{A father's imprint on his daughter's thinking}

Peter McGuffin and Jane Scourfield

$\mathrm{O}$ ne of the puzzles of human gender differences is why boys are more vulnerable than girls to developmental disorders - such as autism - that affect language and social functioning. There has long been a suspicion that this could, in part, be explained by a more general sex difference, whereby women are usually better than men at those aspects of cognition that enable smooth social interactions ('social cognition'). Although such differences might be acquired, and reflect differing male and female social roles, they might also be part of the in-built biological dimorphism that results from the genetic difference between males and females. This intriguing possibility is explored by Skuse and colleagues ${ }^{1}$ on page 705 of this issue, in a study of cognitive function in Turner's syndrome - a sporadic chromosomal disorder in which all (or part) of one X chromosome is missing. Patients have a female appearance and, usually, normal levels of general intelligence, but they have poorly developed secondary sexual characteristics, and short stature.

Normal females have two X chromosomes, and normal males have one $\mathrm{X}$ and one $\mathrm{Y}$ chromosome. But whereas females inherit one $\mathrm{X}$ chromosome from each of their parents, males always receive their $\mathrm{X}$ chromosome from their mother (Fig. 1). This means that, as well as the different chromosomal constitution, there is another possible source of male-female differences resulting from the phenomenon known as genomic imprinting ${ }^{2}$. This refers to the finding that the same genes can result in different phenotypes (expressed characteristics) depending on whether they are inherited from the mother or from the father.

Although the mechanism of imprinting is not fully understood, part of the process involves methylation of DNA, which can effectively result in a gene from one or other parent being 'switched off'. To date there have been no reports of imprinting on the human X chromosome, but the phenome-

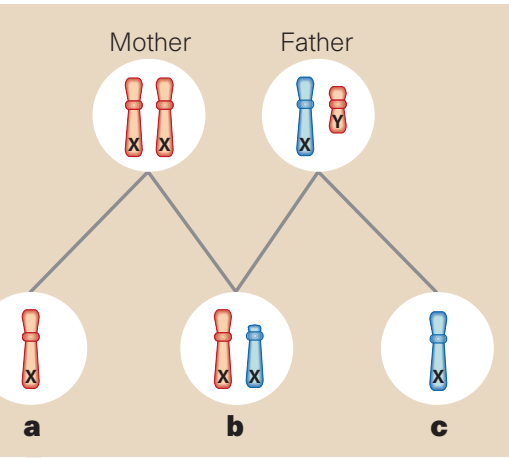

Figure 1 Females with Turner's syndrome have only one complete $\mathrm{X}$ chromosome, which may have come either from their mother (a) or from their father (c). They may also have deletions of part of the $\mathrm{X}$ chromosome, for example the short arm of the paternally derived chromosome (b). Skuse et al. ${ }^{1}$ studied these patients, and showed that Turner's syndrome females with a paternally derived $\mathrm{X}$ chromosome $\left(\mathrm{X}^{\mathrm{P}}\right)$ have much better social skills than those with a maternally derived $\mathrm{X}\left(\mathrm{X}^{\mathrm{m}}\right)$. They conclude that social functioning is influenced by an imprinted gene on the $\mathrm{X}$ chromosome that is switched off when the gene is inherited from the mother. And they believe that this may explain male-female differences, such as why men (who only have a maternally derived $\mathrm{X}$ chromosome) are more susceptible to developmental disorders that affect language and social functioning.

non does occur in other mammals such as the mouse ${ }^{3}$. Skuse et al. ${ }^{1}$ have now tested the hypothesis that imprinting occurs at a gene that influences social cognition by comparing women with Turner's syndrome, whose single $\mathrm{X}$ chromosome came from their father, with those whose $\mathrm{X}$ chromosome was derived from their mother. In doing so, they have identified the first known case of imprinting on the human X chromosome.

Despite having normal intelligence, females with Turner's syndrome tend to show social difficulties ${ }^{4}$ - among the Turner's syndrome girls of school age who were studied by Skuse et al., many had been 'statemented' (the official British system of noting special educational needs). But considerably more of the girls whose $\mathrm{X}$ chromosome was maternally derived $\left(\mathrm{X}^{\mathrm{m}}\right)$ were in this category than those who had received their X chromosome from their father $\left(\mathrm{X}^{\mathrm{p}}\right)$. Moreover, the $\mathrm{X}^{\mathrm{m}}$ girls were judged to have more clinically significant social difficulties, such as offensive or disruptive behaviour, than the $\mathrm{X}^{\mathrm{p}}$ girls or normal males and females.

The differences were explored further using a battery of psychological tests, including a newly devised parental-report scale to measure social cognition in their offspring. This showed that the $\mathrm{X}^{\mathrm{P}}$ females had much better social cognition than the $\mathrm{X}^{\mathrm{m}}$ patients, although both groups fared less well than normal (XX) female controls. The $\mathrm{X}^{\mathrm{P}}$ females also had better verbal IQ than the $\mathrm{X}^{\mathrm{m}}$ patients, but the authors suggest that the differences in social cognition and behaviour might be more closely related to subtle differences in cognitive ability that are not normally measured by conventional intelligence tests. In particular, there were significant differences between $\mathrm{X}^{\mathrm{p}}$ and $\mathrm{X}^{\mathrm{m}}$ patients in an attribute called 'behavioural inhibition'. This is measured by a set of tests in which selecting an appropriate response requires the concurrent inhibition or suppression of an inappropriate response. On the behavioural-inhibition task, $X^{\mathrm{p}}$ females had similar average scores to normal females, who in turn scored better than normal males.

Skuse et al. did a further set of experiments on a small group of patients in whom only part of the short arm of the paternally derived X chromosome was missing. In such cases, the partially deleted chromosome is usually inactivated. However, these women obtained similar scores in the social dysfunction tests to the Turner's syndrome $\mathrm{X}^{\mathrm{p}}$ patients. These data indicate that there is an imprinted gene on the $\mathrm{X}$ chromosome that influences social functioning and related cognitive abilities. This gene escapes Xchromosome inactivation, and it is located either on the long arm of the chromosome, or on a small region of the short arm near the centromere. Such a gene can partly explain the male-female differences in social cognition, as well as in susceptibility to developmental disorders such as autism. But it does not provide the whole story, which is likely to involve an interplay between many genes and interacting environmental factors 5 .

This research raises two important issues, both of which are controversial. The first concerns the more general causes of differences in behaviour between the sexes. This includes both normal behaviour, such as different gender roles, and disorders such as depression (more common in women) and alcoholism (more common in men). Throughout most of the second half of this 